\title{
The effect of bowel preparation regime on interfraction rectal filling variation during image guided radiotherapy for prostate cancer
}

\author{
Ali Hosni ${ }^{1}$, Tara Rosewall', Timothy Craig ${ }^{1}$, Vickie Kong ${ }^{2}$, Andrew Bayley ${ }^{1}$, Alejandro Berlin ${ }^{1}$, Robert Bristow ${ }^{1}$, \\ Charles Catton ${ }^{1}$, Padraig Warde ${ }^{1}$ and Peter Chung ${ }^{1 *}$
}

\begin{abstract}
Background: This study aimed to investigate the tolerability and impact of milk of magnesia (MoM) on interfraction rectal filling during prostate cancer radiotherapy.

Methods: Two groups were retrospectively identified, each consisting of 40 patients with prostate cancer treated with radiotherapy to prostate+/-seminal vesicles, with daily image-guidance in 78Gy/39fractions/8 weeks. The first-group followed anti-flatulence diet with MoM started 3-days prior to planning-CT and continued during radiotherapy, while the second-group followed the same anti-flatulence diet only. The rectum between upper and lower limit of the clinical target volume (CTV) was delineated on planning-CT and on weekly cone-beam-CT (CBCT). Rectal filling was assessed by measurement of anterio-posterior diameter of the rectum at the superior and mid levels of CTV, rectal volume (RV), and average cross-sectional rectal area (CSA; RV/length).

Results: Overall 720 images (80 planning-CT and 640 CBCT images) from 80 patients were analyzed. Using linear mixed models, and after adjusting for baseline values at the time of planning- $C T$ to test the differences in rectal dimensions between both groups over the 8-week treatment period, there were no significant differences in RV $(p=0.4)$, CSA ( $p=0.5)$, anterio-posterior diameter of rectum at superior $(p=0.4)$ or mid level of CTV $(p=0.4)$. In the non-MoM group; $22.5 \%$ of patients had diarrhea compared to $60 \%$ in the MoM group, while $40 \%$ discontinued use of MoM by end of radiotherapy.

Conclusion: The addition of MoM to antiflatulence diet did not reduce the interfraction variation in rectal filling but caused diarrhea in a substantial proportion of patients who then discontinued its use.
\end{abstract}

Keyword: Bowel preparation, Laxative, Prostate cancer, Radiotherapy, Rectum

\section{Background}

Advances in radiotherapy (RT) technology have permitted dose escalation in prostate cancer to improve biochemical control [1]. Precision of RT delivery is an essential component to improve outcomes and reduce associated treatment toxicity [2]. Prostate motion is mainly attributable to changes in rectal volume and shape [3, 4], this has led to various strategies to reproduce consistent rectal filling and

\footnotetext{
* Correspondence: Peter.Chung@rmp.uhn.on.ca

'Department of Radiation Oncology, Princess Margaret Cancer Centre/ University of Toronto, 610 University Ave, Toronto, ON M5G 2M9, Canada

Full list of author information is available at the end of the article
}

provide increased accuracy of RT delivery for prostate cancer.

It has been suggested that using a rectal balloon to achieve reproducible large rectum is one way to reduce variations in rectal filling, thereby reducing prostate motion [5]. Other non-invasive strategies use a rectumemptying approach, by means of laxatives, anti-flatulence diet [6], bowel relaxant [4], probiotics [7], enemas [8], rectum-emptying tube [9], self evacuation [10], or combination of these. However the degree of effectiveness of each of these methods and identification of the most successful approach is still debatable. 
Since 1997, our institutional policy to reduce rectal variation consisted of a defined bowel regimen of an anti-flatulence diet and milk of magnesia (MoM). Nonetheless, subsequent studies using magnesium laxatives failed to show clinically relevant reduction of prostate motion, with high probability of less laxative intake in response to diarrhea [11-14]. Subsequently, our institutional practice changed in 2012 to simple dietary advice (anti-flatulence diet only) without the use of MoM. Although previous investigation had found no reduction in intrafraction prostate motion when using our bowel regimen (with MoM) [12], the efficacy for interfraction rectal filling was not evaluated. Therefore, in the present study we investigated the impact of MoM on the interfraction differences in rectal filling and assessed its tolerability.

\section{Methods}

\section{Patient selection}

Following institutional research ethics board approval, two sequential groups of localized prostate cancer patients treated with volumetric-modulated-arch-therapy (VMAT) to the prostate $+/$ - seminal vesicles (SV) were retrospectively identified. Our institutional practice changed to simple dietary advice without the use of MoM in 2012, so each group consisted of randomly chosen 40 consecutive patients treated in 2011 (MoM cohort) and 2013 (non-MoM cohort). Exclusion criteria were: prostate cancer patients received palliative or postoperative radiotherapy or brachytherapy, pelvic lymph node involvement or distant metastasis, patients with inflammatory bowel disease or taking laxatives, stool softeners or anti-flatulence drugs for other indications.

\section{Bowel regimen}

All patients participated in a routine educational session with a radiation therapist regarding the bladder and rectal preparation for radiotherapy planning and treatment. Patients in the MoM cohort received instructions to follow a bowel regimen which combined an anti-flatulence diet (Table 1) and MoM, while the non-MoM cohort followed the same anti-flatulence diet only. All patients were instructed to start the anti-flatulence diet +/- MoM three days before the planning CT scan and continue

Table 1 Instructions for anti-flatulence diet

\begin{tabular}{ll}
\hline Types of food & Specific foods to avoid \\
\hline Vegetables & $\begin{array}{l}\text { Peas, beans, lentils, broccoli, cauliflower, } \\
\text { brussel sprouts, cabbage, sauerkraut, } \\
\text { cucumber, turnip, rutabaga, onions, garlic }\end{array}$ \\
Fruits & Apples, bananas, prunes, melons \\
High-fat foods & Pastries, pies, deep-fried foods \\
Carbonated drinks & Soda, beer \\
\hline
\end{tabular}

during RT. The initial once a day (bedtime) dose of MoM was $30 \mathrm{~cm}^{3}$, adjusted from 15 to $60 \mathrm{~cm}^{3}$ to achieve a soft bowel movement each morning and stopped in case of lower gastrointestinal (GI) toxicity (i.e. diarrhea). Bowel habit description and daily intake of MoM at baseline and weekly during RT were prospectively documented in the electronic medical record, as a standard of care. Lower GI toxicity (diarrhea during RT) was graded according to RTOG acute toxicity scoring criteria.

\section{Radiotherapy}

Clinical target volume (CTV) included the prostate; while base of the SV was included in the CTV if the risk of SV involvement was $>15 \%$ [15]. Planning target volume (PTV) was created by expansion of the CTV by $10 \mathrm{~mm}$ in all directions, except $7 \mathrm{~mm}$ posteriorly. RT was delivered using VMAT to a prescribed dose of 78Gy in 39 fractions over 8 weeks, using daily prostatefocused image guidance with cone beam CT (CBCT). All patients were treated in the supine position, without rigid immobilization.

\section{Rectal motion assessment}

For each patient, the outer rectal wall was delineated as a solid structure between the upper and lower limits of the CTV, as changes in rectal diameter at this level would likely have the greatest influence on prostate position. This was performed by a single observer on the planning CT and on eight randomly selected CBCTs (one from each week of RT). Rectal filling was assessed by measurement of the anterio-posterior diameter of the rectum at the superior and mid levels of CTV, and by calculation of rectal volume (RV) and the average crosssectional rectal area (CSA; defined as the rectal volume divided by rectal craniocaudal length).

\section{Statistical analyses}

Descriptive statistics were used to describe patient and treatment characteristics. Student's $t$-test was used for comparison of continuous variables. Changes in anterioposterior diameter of the rectum at the superior and mid levels of CTV, RV and CSA between the planning CT and weekly CBCT were compared between both groups by repeated measures analysis using linear mixed models. All tests were two-sided. Statistical analyses were performed using SAS system (version 9.4; SAS Institute Inc, Cary, NC).

\section{Results}

\section{Patient characteristics}

All 80 patients completed the intended course of RT as planned (78Gy over 39 fractions). The characteristics of the patients in both groups are summarized in Table 2. 
Table 2 Patient characteristics in MoM and non-MoM groups

\begin{tabular}{lll}
\hline & MoM group & Non-MoM group \\
& $N=40$ & $N=40$ \\
\hline Age (years) & 72 & 71 \\
- Median & $65-84$ & $60-82$ \\
- Range & & \\
T-category & 22 & 24 \\
- T1 & 18 & 16 \\
- T2 & & \\
Combined Gleason score & 4 & 5 \\
- 6 & 36 & 35 \\
- 7 & & \\
PSA & 8 & 7 \\
- Median & $4-17$ & $5-17$ \\
- Range & & \\
CTV volume $\left(\mathrm{cm}^{3}\right)$ & 45 & $21-139$ \\
- Median & $19-116$ & \\
- Range &
\end{tabular}

MoM milk of magnesia, CTV clinical target volume

No patients received androgen deprivation treatment. The 640 CBCTs selected from the 80 patients were reviewed, and confirmed satisfactory visualisation of the bladder, prostate and seminal vesicles with good definition of rectal boundaries between the upper and lower levels of the CTV.

\section{Interfraction rectal filling characteristics}

In each group, a total of 360 images, including 40 planning CT and 320 CBCT images from the 40 patients were analyzed. Summary of descriptive statistics of rectal volume, average CSA, anterioposterior diameter of the rectum at superior and mid level of CTV in both cohorts at the time of planning CT are shown in Table 3.

The mean RV for MoM vs. non-MoM groups were $34.1+/-21.9$ vs. $35.5+/-15.5 \mathrm{~cm}^{3}$, and the average CSA were $6.3+/-3.7$ vs. $6.7+/-2.4 \mathrm{~cm}^{2}$, while the mean anterioposterior diameter of the rectum at superior and mid level of CTV were $3.2+/-1.1$ vs. $3.4+/-1 \mathrm{~cm}$ and $3.2+/-1$ vs. $3.2+/-0.9 \mathrm{~cm}$ respectively. Using linear mixed models, and after adjusting for baseline values at the time of planning $\mathrm{CT}$ to test the differences in rectal dimensions between both groups over the 8-week treatment period, there were no significant differences between MoM vs. non-MoM group either for RV $(p=0.4)$, average CSA $(p=0.5)$, anterioposterior diameter of the rectum at superior level of CTV $(p=0.4)$ or anterioposterior diameter of rectum at mid level of CTV $(p=0.4)$ (Fig. 1).

\section{MoM tolerability and gastrointestinal toxicity}

In the MoM group, the median volume of MoM taken by patients was $30 \mathrm{~cm}^{3}$ (range, $15-45 \mathrm{~cm}^{3}$ ) in the first week and $15 \mathrm{~cm}^{3}$ (range, $0-30 \mathrm{~cm}^{3}$ ) in the last week. The proportion of patients who took MoM decreased from $100 \%$ in the first week to $60 \%$ in the last week (Fig. 2). Acute RTOG lower GI toxicity in MoM vs. nonMoM groups consisted of G2 diarrhea in 3 patients (7.5\%) vs. 2 patients (5\%) and G1 diarrhea in 21 patients $(52.5 \%)$ vs. 7 patients (17.5\%). In both groups, the onset of diarrhea was reported in the second week of RT, however with higher probability among patients who took MoM (the number of patients who had G1 diarrhea in the second week of RT in MoM vs. non-MoM group was $9[22.5 \%]$ vs. $5[12.5 \%])$.

\section{Discussion}

This study demonstrated no significant difference between the MoM and non-MoM groups in the interfraction variability of rectal dimensions which could affect the prostate motion including RV, average CSA, anterioposterior diameter of the rectum at superior and mid level of CTV. Furthermore, G1-2 diarrhea was experienced in $24(60 \%)$ patients in the MoM group compared to $9(22.5 \%)$ patients who didn't receive MoM, with 16 (40\%) patients discontinuing the use of MoM by the end of radiation treatment.

Image guided radiotherapy (IGRT) is implemented to improve the accuracy of treatment delivery, however it remains difficult to correct for deformation and rotation of the prostate which is mostly influenced by changes in rectal filling. Previous studies have shown that changes in rectal filling can lead to poor outcomes following RT. $[16,17]$ Furthermore, maintaining consistent rectal filling leads to reduction of the required PTV margins [18], which enables dose escalation of RT to the prostate with probability of better tumour control [19], without increasing treatment toxicity thereby improving the therapeutic ratio.

Table 3 Descriptive statistics of rectal measurements at the time of planning

\begin{tabular}{lllc}
\hline & MoM cohort & Non-MoM cohort & p-value \\
\hline Mean rectal volume $\left(\mathrm{cm}^{3}\right)$ & $34.8+/-20.7$ & $35+/-18.7$ & 0.9 \\
Mean average cross sectional area $\left(\mathrm{cm}^{2}\right)$ & $6.5+/-3.5$ & $6.5+/-2.6$ & 3.9 \\
Mean anterioposterior diameter at superior level of CTV $(\mathrm{cm})$ & $3.2+/-1$ & $3.2+/-0.8$ & 3.9 \\
Mean anterioposterior diameter at mid level of CTV (cm) & $3.2+/-0.9$ & $3+/-0.9$ \\
\hline
\end{tabular}




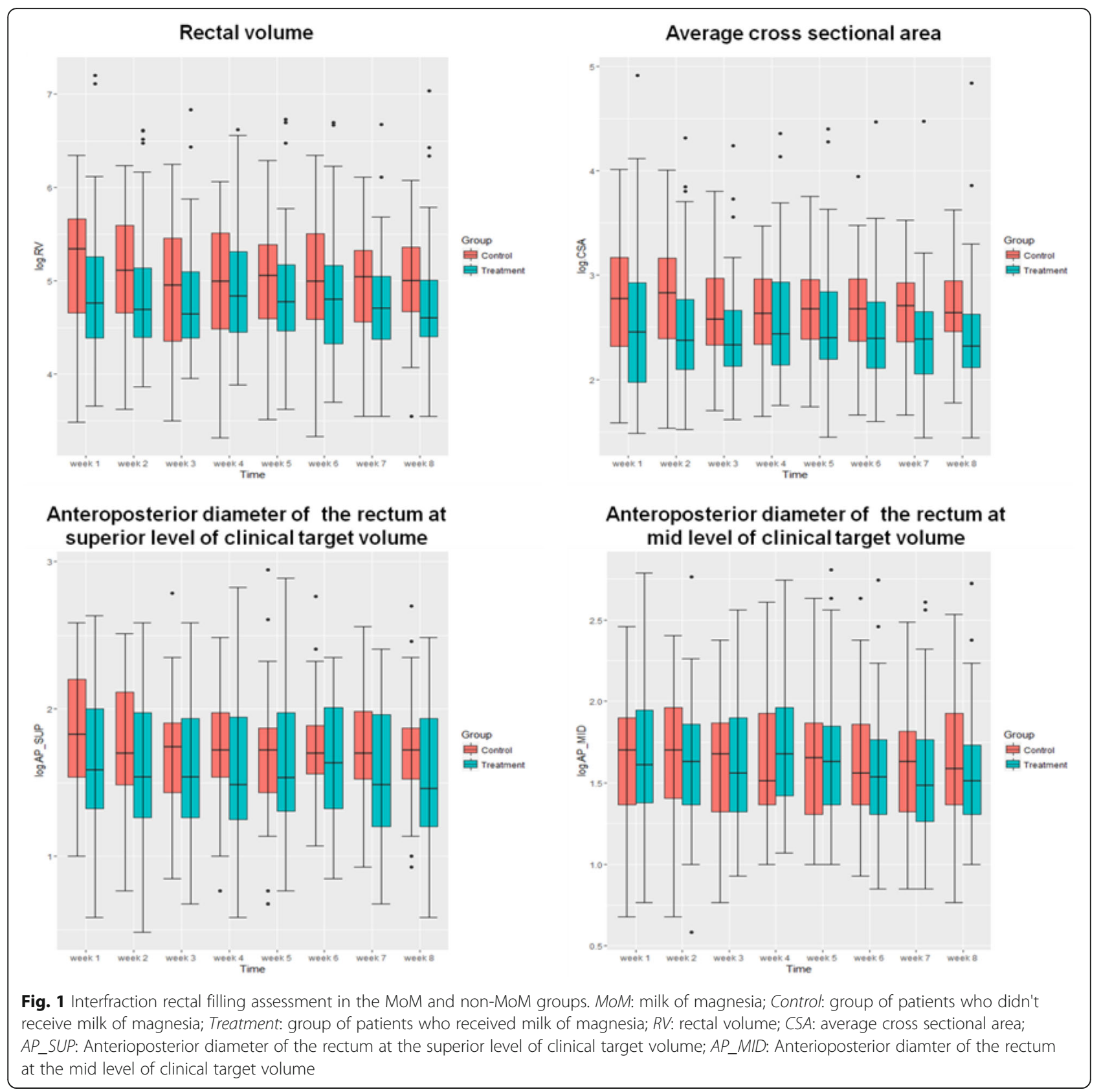

The use of laxatives and anti-flatulence diet to reduce rectal filling variation has been previously investigated. In a randomised controlled trial (RCT) with 30 prostate cancer patients, Oat et al. reported that dietary intervention with psyllium $(20 \mathrm{~g} / \mathrm{d}, n=15)$ didn't significantly reduce the variability in RV or rectal filling at superior level of the prostate. It was however, associated with consistent rectal filling at mid-level of the prostate [20]. In another RCT of prostate cancer patients assigned to receive magnesium oxide (500 $\mathrm{mg}$ twice a day, $n=46$ ) or placebo $(n=46)$ during RT and similar to our results, there was no significant difference in RV between the treatment arms and magnesium oxide was not effective in reducing the interfraction rectal filling [14]. Furthermore, several other studies using magnesium laxatives were unable to show a clinically relevant reduction of inter- or intra-fractional prostate motion [11-13].

Despite an inability to reduce interfraction prostate motion, a dietary protocol with laxatives may potentially decrease the rectal distention related to gas resulting in better CBCT image quality and facilitating the IGRT process [11]. On the other hand, tolerability of the laxative remains an important clinical consideration, with higher probability of less laxative intake or even anti- 


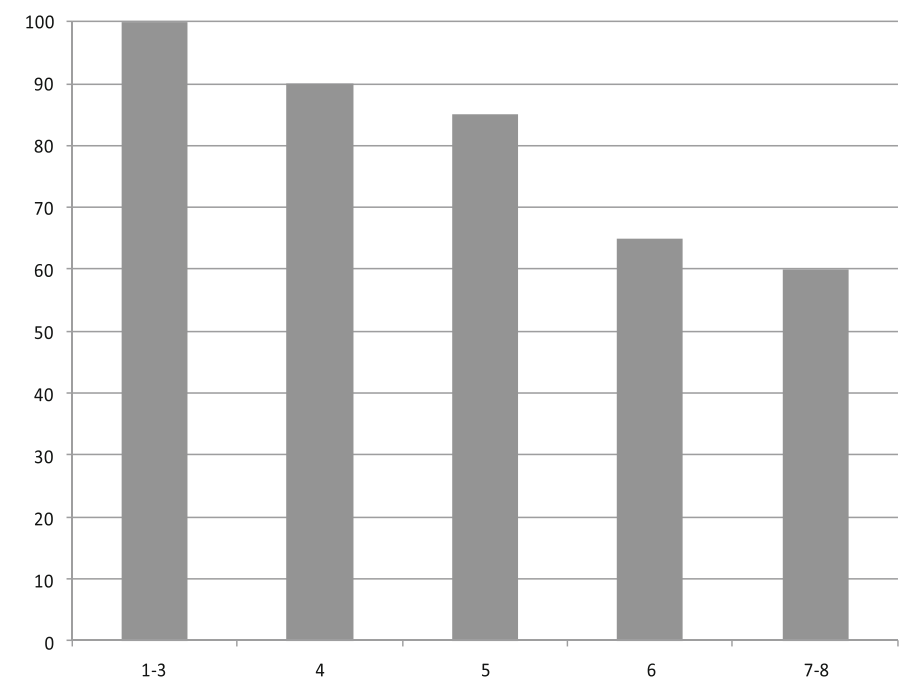

Fig. 2 Proportion of patients who took milk of magnesia during radiotherapy

diarrheal use in response to more frequent bowel movement or changing the stool texture [12,13]. In a RCT of magnesium oxide $(n=46)$ vs. placebo $(n=46)$, patients in the intervention arm had more frequent grade $\geq 2$ acute GI toxicity (37\% vs. $22 \%$ ) and were more often prescribed anti-diarrheal medicines during RT (15\% vs. 9\%) [13]. We had previously reported that the proportion of patients who didn't take MoM increased from $8 \%$ in the first week to $44 \%$ in the last week of RT [12]. Consistently, in the current study, $60 \%$ of patients who took MoM had diarrhea, and $40 \%$ discontinued its use by the end of radiation treatment.

The findings from this study should be interpreted in context with its methodological limitations. The most significant limitation is related to its retrospective and non-randomized nature which may have led to undocumented differences between the cohorts which may have masked the effect of diet and MoM. Also, compliance with the antiflatulence diet was not quantified and may have been different between the two groups, which may have negated the effect of the diet on interfraction rectal filling variation. Nonetheless, when considered in context with previous evaluations of this subject, our results confirm that the addition of MoM to an antiflatulence diet does not lead to more consistent rectal filling. Variations in rectal volume and size can influence both translational prostate motion (which can be corrected with IGRT), and rotational prostate motion (which is more difficult to mitigate by current state-of-the-art technologies). However, rectal filling is not the sole factor with potential impact in prostate spatial localization, and different intervention approaches considering altogether the impact of rectal and bladder filling, patient positioning and breathing on prostate motion should be investigated.

\section{Conclusion}

The addition of MoM to an antiflatulence diet did not reduce interfraction variation in rectal filling and may cause diarrhea resulting in a substantial proportion of patients discontinuing its use. Simple dietary instructions appeared to be just as effective at reducing interfraction rectal variability, and MoM should be omitted from routine use during prostate radiotherapy.

\section{Abbreviations}

CBCT: Cone beam CT; CSA: Cross sectional area; CT: Computed tomography; CTV: Clinical target volume; GI: Gastrointestinal; IGRT: Image guided radiotherapy; MoM: Milk of magnesia; PTV: Planning target volume; RCT: Randomized controlled trial; RT: Radiotherapy; RV: Rectal volume; SV: Seminal vesicles; VMAT: Volumetric modulated arch therapy.

\section{Acknowledgements}

None.

Funding

None.

\section{Availability of data and material}

The datasets analysed during the current study available from the corresponding author on reasonable request.

\section{Authors' contributions}

$\mathrm{AH}, \mathrm{TR}, \mathrm{TC}, \mathrm{VK}$ and $\mathrm{PC}$ contributed to the conception and design of the study, development of methodology and acquisition of data. All authors contributed to analysis and interpretation of data, and writing, review and final approval of the manuscript.

\section{Competing interests}

The authors declare that they have no competing interests.

\section{Consent for publication}

Not applicable.

\section{Ethics approval and consent to participate}

This study was initiated after the approval of the institutional research ethics board review at the University Health Network of the study protocol \#14-8278-CE.

\section{Presentation}

Presented in part as an abstract at annual meeting of ASTRO 2015. 


\section{Author details}

'Department of Radiation Oncology, Princess Margaret Cancer Centre/ University of Toronto, 610 University Ave, Toronto, ON M5G 2M9, Canada. ${ }^{2}$ Radiation Medicine Program, Princess Margaret Cancer Centre, Toronto, ON, Canada.

Received: 15 August 2016 Accepted: 22 February 2017

Published online: 09 March 2017

\section{References}

1. Zelefsky MJ, Pei X, Chou JF, et al. Dose escalation for prostate cancer radiotherapy: predictors of long-term biochemical tumor control and distant metastases-free survival outcomes. Eur Urol. 2011:60:1133-9.

2. Zelefsky MJ, Kollmeier M, Cox B, et al. Improved clinical outcomes with high-dose image quided radiotherapy compared with non-IGRT for the treatment of clinically localized prostate cancer. Int J Radiat Oncol Biol Phys. 2012:84:125-9.

3. Zelefsky MJ, Crean D, Mageras GS, et al. Quantification and predictors of prostate position variability in 50 patients evaluated with multiple CT scans during conformal radiotherapy. Radiother Oncol. 1999;50:225-34.

4. Padhani AR, Khoo VS, Suckling J, Husband JE, Leach MO, Dearnaley DP. Evaluating the effect of rectal distension and rectal movement on prostate gland position using cine MRI. Int J Radiat Oncol Biol Phys. 1999;44:525-33.

5. Smeenk RJ, Teh BS, Butler EB, van Lin EN, Kaanders JH. Is there a role for endorectal balloons in prostate radiotherapy? a systematic review. Radiother Oncol. 2010:95:277-82.

6. McNair HA, Wedlake L, McVey GP, Thomas K, Andreyev J, Dearnaley DP. Can diet combined with treatment scheduling achieve consistency of rectal filling in patients receiving radiotherapy to the prostate? Radiother Oncol. 2011;101:471-8.

7. Ki Y, Kim W, Nam J, et al. Probiotics for rectal volume variation during radiation therapy for prostate cancer. Int J Radiat Oncol Biol Phys. 2013;87: $646-50$

8. Villeirs GM, De Meerleer GO, Verstraete KL, De Neve WJ. Magnetic resonance assessment of prostate localization variability in intensity-modulated radiotherapy for prostate cancer. Int J Radiat Oncol Biol Phys. 2004;60:1611-21.

9. Fuji H, Murayama S, Niwakawa M, et al. Changes in rectal volume and prostate localization due to placement of a rectum-emptying tube. Jpn J Radiol. 2009;27:205-12.

10. Ogino I, Uemura H, Inoue T, Kubota Y, Nomura K, Okamoto N. Reduction of prostate motion by removal of gas in rectum during radiotherapy. Int J Radiat Oncol Biol Phys. 2008;72:456-66.

11. Smitsmans MH, Pos FJ, de Bois J, et al. The influence of a dietary protocol on cone beam CT-guided radiotherapy for prostate cancer patients. Int J Radiat Oncol Biol Phys. 2008;71:1279-86.

12. Nichol AM, Warde PR, Lockwood GA, et al. A cinematic magnetic resonance imaging study of milk of magnesia laxative and an antiflatulent diet to reduce intrafraction prostate motion. Int J Radiat Oncol Biol Phys. 2010;77: 1072-8.

13. Lips IM, van Gils $\mathrm{CH}$, Kotte AN, et al. A double-blind placebo-controlled randomized clinical trial with magnesium oxide to reduce intrafraction prostate motion for prostate cancer radiotherapy. Int J Radiat Oncol Biol Phys. 2012;83:653-60.

14. den Harder AM, van Gils CH, Kotte AN, van Vulpen M, Lips IM. Effect of magnesium oxide on interfraction prostate motion and rectal filling in prostate cancer radiotherapy: analysis of a randomized clinical trial. Strahlentherapie und Onkologie : Organ der Deutschen Rontgengesellschaft ... [et al]. 2014;190:758-761.

15. Partin AW, Kattan MW, Subong EN, et al. Combination of prostate-specific antigen, clinical stage, and Gleason score to predict pathological stage of localized prostate cancer. A multi-institutional update. JAMA. 1997:277:1445-51.

16. Heemsbergen WD, Hoogeman MS, Witte MG, Peeters ST, Incrocci L, Lebesque $\mathrm{JV}$. Increased risk of biochemical and clinical failure for prostate patients with a large rectum at radiotherapy planning: results from the Dutch trial of $68 \mathrm{GY}$ versus 78 Gy. Int J Radiat Oncol Biol Phys. 2007;67:1418-24.

17. de Crevoisier R, Tucker SL, Dong L, et al. Increased risk of biochemical and local failure in patients with distended rectum on the planning $C T$ for prostate cancer radiotherapy. Int J Radiat Oncol Biol Phys. 2005;62:965-73.

18. Litzenberg DW, Balter JM, Hadley SW, et al. Influence of intrafraction motion on margins for prostate radiotherapy. Int J Radiat Oncol Biol Phys. 2006;65: 548-53.
19. Viani GA, Stefano EJ, Afonso SL. Higher-than-conventional radiation doses in localized prostate cancer treatment: a meta-analysis of randomized, controlled trials. Int J Radiat Oncol Biol Phys. 2009;74:1405-18.

20. Oates RW, Schneider ME, Lim Joon M, et al. A randomised study of a diet intervention to maintain consistent rectal volume for patients receiving radical radiotherapy to the prostate. Acta Oncol. 2014;53:569-71.

\section{Submit your next manuscript to BioMed Central and we will help you at every step:}

- We accept pre-submission inquiries

- Our selector tool helps you to find the most relevant journal

- We provide round the clock customer support

- Convenient online submission

- Thorough peer review

- Inclusion in PubMed and all major indexing services

- Maximum visibility for your research

Submit your manuscript at www.biomedcentral.com/submit
Biomed Central 